\title{
Metacognition and Causal Inference in Audiovisual Speech
}

\author{
Faith Kimmet ${ }^{1}$, Samantha Pedersen ${ }^{1}$, Victoria Cardenas ${ }^{1}$, Camila Rubiera ${ }^{1}$, Grey Johnson ${ }^{1}$, \\ Addison Sans ${ }^{1}$, and Brian Odegaard ${ }^{1}$ \\ ${ }^{1}$ University of Florida
}

\section{Author Note}

Faith Kimmet, Camila Rubiera, Samantha Pedersen, Victoria Cardenas, Grey Johnson, and Addison Sans do not currently have ORCIDs.

Brian Odegaard: https://orcid.org/0000-0002-5459-1884

Brian Odegaard is at the University of Florida in Gainesville, FL.

We have no conflicts of interest to disclose.

Correspondence concerning this article should be addressed to Brian Odegaard, 945 Center Dr, Gainesville, FL 32603, Email: bodegaard@ufl.edu 


\section{Summary}

In multisensory environments, our brains perform causal inference to estimate which sources produce specific sensory signals. Decades of research has revealed the dynamics which underlie this process of causal inference for multisensory (audiovisual) signals, including how temporal, spatial, and semantic relationships between stimuli influence the brain's decision about whether to integrate or segregate. However, presently, very little is known about the relationship between metacognition and multisensory integration, and the characteristics of perceptual confidence for audiovisual signals. In this investigation, we ask two questions about the relationship between metacognition and multisensory causal inference: are observers' confidence ratings for judgments about congruent, McGurk, and rarely integrated speech similar, or different? And do confidence judgments distinguish between these three scenarios when the perceived syllable is identical? To answer these questions, 92 online participants completed experiments where on each trial, participants reported which syllable they perceived, and rated confidence in their judgment. Results from Experiment 1 showed that confidence was highest for congruent speech and lower for McGurk and rarely integrated speech. In Experiment 2, when the perceived syllable for congruent and McGurk videos was matched, confidence scores were higher for congruent stimuli compared to McGurk stimuli. In Experiment 3, when the perceived syllable was matched between McGurk and rarely integrated stimuli, confidence judgments were similar between the two conditions. Together, these results provide evidence of the capacities and limitations of metacognition's ability to index multisensory causal inference.

Keywords: Metacognition, Causal Inference, Speech, Multisensory, McGurk 


\section{Introduction}

Our brains quickly and effortlessly integrate incongruent audiovisual sensory signals to produce a coherent perception of the world. For example, the presentation of incongruent numbers of brief flashes and beeps often yields illusory perception of phantom flashes (Shams et al., 2000). Simultaneous, spatially discrepant audiovisual signals can cause the auditory component to be mislocalized close to where the visual component occurred (Pick et al., 1969; Welch and Warren, 1980). And incongruent audiovisual speech syllables (e.g., Visual "Ba" and auditory "Ga") can produce perception of a unique, third syllable (e.g., "Da") (McGurk and MacDonald, 1976). These examples of multisensory integration are often referred to as "illusions" in the scientific literature (Stevenson et al., 2012) and reflect a general principle: when sensory cues are in conflict with one another, our brains have specific mechanisms which reconcile differences to produce unified, integrated perception (Ernst and Banks, 2002; Knill and Richards, 1996; Körding et al., 2007).

While behavioral and computational studies of these multisensory illusions abound, little is known about the relationship between metacognition and multisensory integration. Metacognition can be generally defined as "thinking about thinking" (Flavell, 1979); in perceptual paradigms, metacognition can be measured by obtaining confidence ratings in perceptual decisions (Fleming and Lau, 2014). While the study of visual metacognition is well-established (Rahnev et al., 2021), research on the relationship between multisensory integration and metacognition is limited to only a few studies (White et al., 2014), and how metacognitive judgments interact with the process of "causal inference" that influences sensory integration remains almost entirely unexplored (Deroy et al., 2016; Shams and Beierholm, 2010, 2021). In this investigation, we ask the following question: does the average level of confidence differ for sensory information that is integrated from discrepant sources, segregated from multiple sources, or arises from only a single source? And more specifically, if the reported percept 
across different scenarios is the same, does the average level of confidence distinguish between congruent speech, integrated speech, and segregated speech?

Answering these questions is critical to better understand the relationship between metacognition and the hierarchical computations that form the basis of all sensory experiences, including multisensory integration (Körding et al., 2007; McGovern et al., 2016; Rohe et al., 2019; Rohe and Noppeney, 2015). Currently, multisensory integration is thought to reflect the principles of Bayesian causal inference, as audiovisual cues are integrated or segregated based on a combination of noisy sensory encoding and priors that govern perception of a common cause (Magnotti et al., 2018; Odegaard et al., 2015; Rohe and Noppeney, 2015). However, it is currently unknown whether our metacognitive awareness of this process is limited to the integrated end product of the sensory inference, or whether it can distinguish between congruent, incongruent, and illusory sensory content produced by various causal structures (Figure 1). Previous research provides preliminary evidence that metacognition may indeed be able to distinguish among different causal scenarios (White et al., 2014), but supporting data is sparse, and more research is needed.
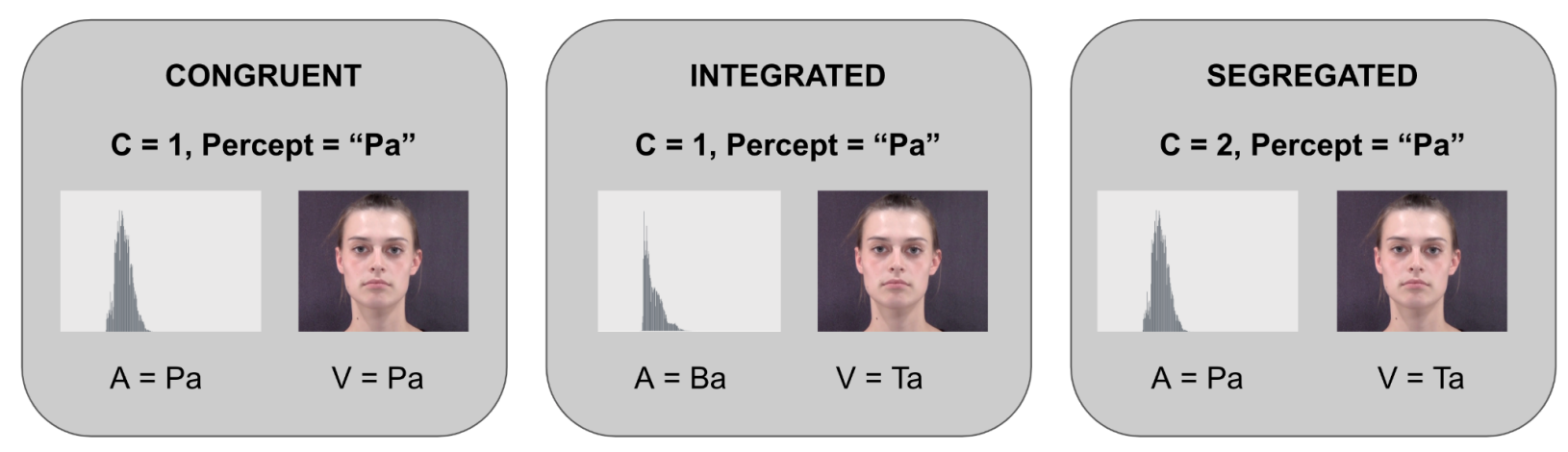

Figure 1. Different audiovisual speech syllable combinations can yield identical reported percepts. In the standard McGurk paradigm (McGurk and MacDonald, 1976), an observer views a short audiovisual video which contains an auditory syllable and a visible person (or mouth) pronouncing a single syllable. This paradigm can result in three scenarios: congruent speech, if the auditory and visual syllables are identical (left panel); integrated speech, if the auditory and visual syllables differ and cause perception of an intermediate, third syllable (middle panel); and segregated speech, where syllables differ enough that they are perceived as causally separate, and the participant most often reports perceiving the auditory syllable (right panel). Interestingly, these three scenarios can potentially all give rise to the same 
perceived syllable ("Pa"), as in the example shown here. This process of inference has been accounted for by Bayesian models (Körding et al., 2007; Magnotti et al., 2013, 2018; Magnotti and Beauchamp, 2017; Shams and Beierholm, 2010) which assume that observers have access to noisy sensory representations, but must infer the underlying causes of information which generated the signals. In the McGurk task, the number of causes can either be one $(C=1)$ or two $(C=2)$. In this investigation, we ask three questions: whether metacognition can differentiate between congruent, integrated, and segregated speech with unmatched reports (Experiment 1), whether metacognition can distinguish between congruent and integrated scenarios where the reported percept is the same and the underlying causal structure is the same $(\mathrm{C}=1)$ (Experiment 2), and whether metacognition can distinguish between scenarios where the reported percept is the same, but the underlying causal structure is different (i.e., $\mathrm{C}=1$ and $\mathrm{C}=2)($ Experiment 3$)$.

In the present study, we addressed this topic in experiments which assess metacognitive judgments for audiovisual McGurk speech stimuli. In our first experiment, observers viewed nine different videos from three mutually exclusive categories: stimuli that are rarely integrated, stimuli that often produce a McGurk illusion, and fully congruent audiovisual stimuli. On each trial, they rated confidence from 0 (not at all confident) to 100 (extremely confident) in their perceptual judgment. Our hypothesis was that observers would display the lowest confidence in illusory McGurk trials, the highest confidence in fully congruent audiovisual speech, and intermediate confidence in syllable combinations that are rarely integrated. In Experiment 2, we implemented a new task with 12 different audiovisual videos; our main analysis involved selecting pairs of trials where the integrated McGurk percept matched a particular type of congruent audiovisual trial (e.g., visual Ba/auditory Ga causes an observer to perceive "Da," and visual Da/auditory Da causes an observer to perceive "Da"). We hypothesized that observers would be more confident in fully congruent trials compared to McGurk trials, even when these trials resulted in the identical reports. In our third experiment, we evaluated whether confidence judgments would distinguish between scenarios where the reported percept was the same, but the underlying causal structure of the stimuli was different. Our hypothesis was that, on average, subjects would express higher confidence in stimuli that were rarely integrated, as these percepts would likely be less ambiguous than McGurk percepts. 
As anticipated, results from the first experiment revealed that confidence was highest on congruent speech, intermediate for rarely-integrated incongruent speech, and lowest for McGurk speech, demonstrating that metacognition differs across these causal scenarios. In our second experiment, we showed that even when the perceived syllable was the same, confidence was higher for audiovisual congruent speech compared to McGurk speech. This indicates that even when multisensory percepts are identical (Deroy et al., 2016; Freeman and Simoncelli, 2011), metacognition can distinguish congruent and integrated speech. In our third experiment, when the perceived syllable was matched between McGurk and rarely integrated stimuli, confidence only differed between the two conditions for one set of stimuli, and no clear trend emerged across all conditions. Together, these results indicate that higher-order metacognitive systems can distinguish different sources of multisensory information in some, but not all, circumstances.

\section{Experiment 1 - Method}

In Experiment 1, we explored whether confidence judgments for audiovisual speech

would differ across conditions with congruent audiovisual syllables, McGurk syllables, and rarely integrated audiovisual syllable combinations.

\section{Participants}

Thirty-five participants enrolled in this online experiment, which was coded in jsPsych 6.3.1 (de Leeuw, 2015), and launched using custom code through Google Drive's Application Programming Interface (API). Participants were recruited through Prolific.co, and were awarded $\$ 4.30$ upon completion of the task. Three participants completed less than half of the task and were excluded from further analysis; additionally, two participants refreshed their browser window while completing the experiment and completed most of the task twice, so they were also excluded. Thus, 30 participants were included in our final analysis (18 men, 12 women; mean age $=32.8$ years $)$. 


\section{Stimuli}

For our McGurk stimuli, we selected video and audio clips from the Oldenburg Audio Visual Speech Stimuli (OLAVS) set (Stropahl et al., 2017) and created nine audiovisual combinations. Our reason for using these specific stimuli was to have a balanced stimulus design, with three fully-congruent audiovisual stimulus pairs (auditory Ba/visual Ba; auditory Ma /visual Ma; auditory Pa/visual Pa), three pairs known to produce illusory McGurk syllables (auditory $\mathrm{Ba} /$ visual $\mathrm{Ga}=$ perceive $\mathrm{Da}$ or $\mathrm{Ma}$; auditory $\mathrm{Pa} /$ visual $\mathrm{Na}=$ perceive $\mathrm{Ka}$ or $\mathrm{Ta}$, and auditory Ma/visual $\mathrm{Ta}=$ perceive $\mathrm{Na}$ or $\mathrm{La}$ ), and three pairs of incongruent stimuli that are rarely integrated (auditory Da/visual Ma; auditory $\mathrm{Na}$ /visual Da; auditory Ta/visual $\mathrm{Ga}$ ). All stimuli were from speaker TK01 in the Stropahl et al. (2017) dataset. There were four potential answer options on each trial. For McGurk trials, the possible answer choices corresponded to the visual syllable, the auditory syllable, and two syllables which reflect "fused" percepts (from Table 1 in Stropahl et al., 2017). For rarely integrated trials, the possible answer choices corresponded to the visual syllable, the auditory syllable, and two "foil" answers that did not correspond to any presented or integrated percept. For congruent trials, we selected the correct syllable, as well as three potential foils.

\section{Procedure}

Participants began our online task by enrolling through the website Prolific.co. Following a welcome screen, participants read through our online consent form (IRB \#201902462, University of Florida) and provided consent by checking a box next to the statement, "I agree to participate in this study." Next, participants reported their sex, age, and viewed a photograph which demonstrated roughly how far they should be from the screen while participating in the experiment. Participants were then presented with a sample McGurk video, and were asked to adjust their speaker volume to a comfortable level. They could press a "repeat" button as many times as necessary to adjust the volume; a "continue" button moved the experiment forward. 
Following this, participants completed nine practice trials. On each practice trial, a video of the speaker was presented for $2000 \mathrm{~ms}$, and then the answer options were shown. Participants provided a categorical answer about the perceived syllable by pushing a button on the screen with their mouse cursor. Next, participants provided an answer about their confidence by moving a slider on the screen on a continuous scale from 0 (no confidence) to 100 (fully confident).

After the practice session, participants began the real experiment which consisted of 3 blocks of 45 trials. As in the practice, on each trial participants were shown an audiovisual video of a speaker, which could contain congruent, McGurk, or rarely integrated audiovisual syllables. Following each video, answer options were shown, which were customized for each specific video type. For example, for congruent stimuli, besides the correct choice, three plausible foils were selected as the other possible answer options. For McGurk stimuli, the four answer options included the visual component of the video, the auditory component of the video, the "Fusion1" responses from Table 1 in Stropahl et al. 2017, and the "Fusion 2" responses from that same table. For rarely integrated stimuli, the four answer options included the visual component, the auditory component, and two foils. The answer option order was randomized on each trial. After they reported the syllable they perceived, participants rated their confidence in each judgment on the scale from 0-100. The slider started from a random position on each trial, and moved in increments of 1 on the scale. Participants were allowed to take breaks between blocks. Upon finishing the task, participants were given a "completion code" to receive payment for their time \& effort.

\section{Results}

We investigated whether metacognitive judgments could distinguish between McGurk speech and other forms of audiovisual speech. Figure 2 presents the average confidence levels across the three conditions in Experiment 1: fully congruent audiovisual syllables, McGurk 
syllables, and rarely integrated audiovisual syllables. Looking at the pattern of results in Figure

1, our primary hypothesis was confirmed: congruent audiovisual speech was rated with the highest amount of confidence (mean $=89.6)$, rarely integrated speech was rated with the second highest amount of confidence (mean $=84.0$ ), and McGurk syllables were rated with the lowest confidence $($ mean $=81.3)$.

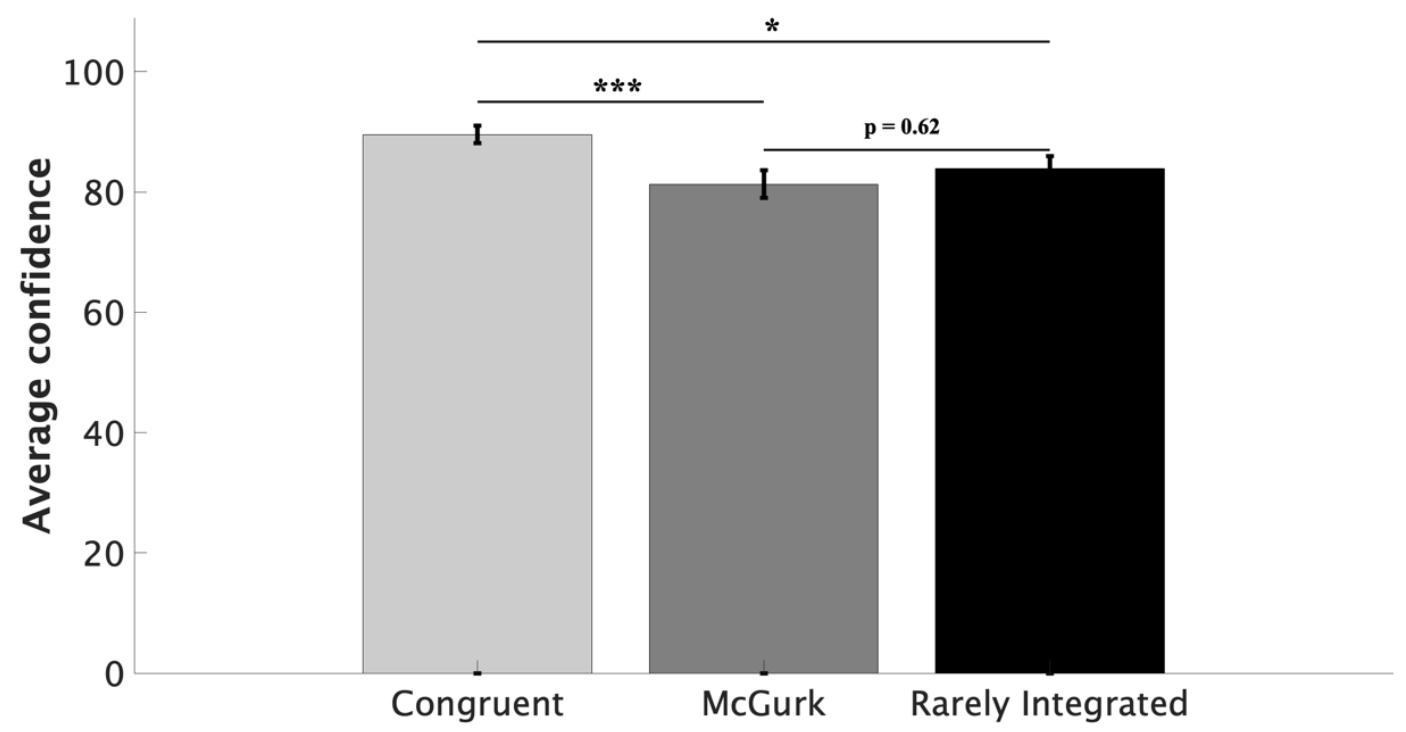

Figure 2. Average confidence ratings (i.e., the average of each subject's average confidence) for judgments about audiovisual speech. In our perceptual task, observers viewed audiovisual videos of speakers pronouncing syllables that were either congruent (e.g., auditory Da-visual Da), specific McGurk combinations of syllables (e.g., auditory Ba- visual Ga), or incongruent combinations of syllables that were rarely integrated (e.g., auditory $\mathrm{Da}$ - visual $\mathrm{Ma}$ ). After making a judgment about the syllable that was perceived, observers were asked to rate their confidence on a scale from 0-100. As shown in the figure, confidence was highest for congruent syllables, lowest for McGurk syllables, and intermediate for rarely integrated audiovisual syllable combinations. Error bars represent standard error of the mean across subjects. ${ }^{* * *} p<.001,{ }^{*} p<.05$

To test these apparent effects, the data were first analyzed using a one-way withinparticipants ANOVA. Mauchly's test indicated that the assumption of sphericity had not been violated, $\mathbf{X}^{2}(2)=3.28, p=.19$; our ANOVA revealed that confidence was significantly different across conditions $(F(29)=10.579, p<0.001)$. To compare individual condition means, multiple t-tests were performed, with Bonferroni correction to maintain an alpha level of .05. The difference between confidence ratings for congruent speech and McGurk speech was very 
strong $\left(\mathrm{t}(29)=5.44, \mathrm{p}_{\text {bonf }}<.001, d=0.99\right)$, and the difference between average confidence for congruent speech and rarely integrated speech was somewhat smaller $\left(\mathrm{t}(29)=2.97, \mathrm{p}_{\mathrm{bonf}}=\right.$ $0.02, d=0.54)$. The difference between confidence for judgments about rarely integrated speech and McGurk speech, however, was not statistically significant $\left(\mathrm{t}(29)=1.29, \mathrm{p}_{\mathrm{bonf}}=0.62\right.$, $d=0.24)$.

As expected, the number of correct judgments (i.e., correctly identifying the auditory component that was included) was different across all three conditions $(F(29)=97.89, p<.001)$, with high accuracy rates for the congruent $(92.7 \%)$ and rarely integrated (92.9\%) conditions, but a low accuracy rate for the McGurk condition (27.9\%). The low correct rate in the McGurk condition was primarily driven by a strong tendency to select one of the two perceptual fusion responses; on McGurk trials, fused responses made up $71.5 \%$ of answers, with responses to the auditory syllable much less frequent (27.9\%) and responses reporting the visual component extremely rare (0.5\%). As expected based on previous research (Stropahl et al., 2017), the fusion rates for the three different McGurk conditions were quite comparable $(F(29)=1.27, p=$ 0.33), with auditory $\mathrm{Ba} /$ visual $\mathrm{Ga}$, auditory $\mathrm{Pa} /$ visual $\mathrm{Na}$, and auditory Ma/visual Ta all yielding comparable rates of fused responses $(72.5 \%, 67 \%$, and $75.3 \%$, respectively).

\section{Experiment 2 - Method}

After demonstrating in Experiment 1 that confidence differed across congruent, McGurk, and rarely integrated speech, in Experiment 2, we aimed to determine whether confidence differed between congruent and illusory speech when the reported percept was identical (Deroy et al., 2016). Previous research has identified pairs of stimuli which produce the same perceptual reports (Freeman and Simoncelli, 2011), but it remains an open question whether metacognitive systems distinguish between different types of multisensory stimuli yielding the same reported percept (Deroy et al., 2016; Shams and Beierholm, 2021). 


\section{Participants}

Forty-two participants enrolled in our second online experiment, which was coded using jsPsych 6.3.1 (de Leeuw, 2015) and Google's web API, and administered through Prolific.co. Participants were awarded $\$ 4.30$ upon completion of the task. Seven participants completed less than half of the task and were excluded from further analysis; one participant refreshed the browser window while completing the experiment and completed most of the task twice, and was also excluded; two other subjects had data packet errors causing mismatches between trials and responses, and were also excluded. Thus, 32 participants were included in our final analysis (12 men, 19 women, 1 unreported); mean age $=29.9$ years)

\section{Stimuli}

For our McGurk stimuli in Experiment 2, we selected video and audio clips from the Oldenburg Audio Visual Speech Stimuli (OLAVS) set (Stropahl et al., 2017) and created twelve audiovisual combinations from Speaker TK01. Our reason for using stimuli from three conditions was to have a balanced stimulus design between fully-congruent syllables, McGurk syllable combinations, and incongruent syllable combinations that are rarely integrated, so that participants would not always be integrating stimuli on every trial. Our fully-congruent audiovisual stimulus pairs included auditory $\mathrm{Na}$ /visual $\mathrm{Na}$, auditory $\mathrm{Pa} /$ visual $\mathrm{Pa}$, auditory Da/visual Da, and auditory Ta/visual Ta. Our McGurk stimulus pairs included auditory Ma/visual $\mathrm{Ta}$, auditory $\mathrm{Ba} /$ visual Ta, auditory $\mathrm{Ba} / \mathrm{visual} \mathrm{Ka}$, and auditory $\mathrm{Pa} / \mathrm{visual} \mathrm{Da}$. Our incongruent pairs that were rarely integrated included auditory $\mathrm{Na} /$ visual $\mathrm{Da}$, auditory $\mathrm{Pa} /$ visual $\mathrm{Ta}$, auditory Ga/visual Ta, and auditory Ta/visual Ma.

Our motivation for selecting these specific stimuli was to create conditions where the perceived syllable could be matched across the congruent and McGurk conditions. For example, the perceived syllable in each of the congruent conditions listed above is, respectively: $\mathrm{Na}, \mathrm{Pa}, \mathrm{Da}$, Ta. After piloting the McGurk conditions, we found that the four McGurk conditions 
described above can also produce (in many individuals) the perceived syllables $\mathrm{Na}, \mathrm{Pa}, \mathrm{Da}$, and Ta. The rarely-integrated cases were included as foils.

\section{Procedure}

Following acceptance of our online consent form, participants completed a "virtual chinrest" test to measure viewing distance, a sound check screen to allow them to adjust volume to a comfortable level, and introductory screens with task instructions. Subjects also reported their age and their biological sex on the introductory screens. Participants then completed three practice trials to acquaint themselves with the primary task. On each practice trial, a given video of a McGurk speaker was presented for approximately $2000 \mathrm{~ms}$. They provided a categorical answer about the perceived syllable by pushing a button on the screen participants provided an answer about their confidence by moving a slider on a continuous scale from 0 (no confidence) to 100 (fully confident).

After the practice session, participants began the real experiment which consisted of 3 blocks of 60 trials. As in the first experiment, participants performed two tasks on each trial: (1) they reported the syllable they perceived after hearing/seeing the audiovisual video, and (2) they rated their confidence in each judgment on the scale from 0-100. Participants were allowed to take breaks between blocks. Upon finishing the task, participants were given a "completion code" to receive payment for their time \& effort.

\section{Results}

We replicated the results from our first experiment, as participants were the most confident in fully congruent audiovisual stimuli, the least confident in McGurk stimuli, and somewhat in between for stimuli that were rarely integrated. To test whether these differences were significant, we first evaluated the assumption of sphericity for our repeated-factor manipulation of stimulus type. Mauchly's test indicated that the assumption of sphericity had been violated, $\mathbf{X}^{2}(2)=13.23, p=.001$, so we used the Greenhouse-Geisser correction for our repeated measures ANOVA, which revealed a significant effect of stimulus type on perceptual 
confidence $(F(1.474)=24.31, p<.001)$. Post-hoc tests revealed that congruent stimuli resulted in significantly higher confidence than rarely integrated stimuli (mean difference $=4.95, t=4.8$, $\mathrm{p}_{\mathrm{bonf}}<.001, d=0.85$ ) or McGurk stimuli (mean difference $=9.98, \mathrm{t}=5.61, \mathrm{p}_{\mathrm{bonf}}<.001, d=0.99$ ). Interestingly, for this set of stimuli, the difference between rarely integrated stimuli and McGurk stimuli was also significant (mean difference $=5.03, \mathrm{t}=3.63, \mathrm{p}_{\mathrm{bonf}}=.003, d=0.64$ ).

Next, we followed with our most important analysis: determining whether confidence judgments could distinguish between congruent videos and McGurk videos when the reported syllable was the same. To perform this analysis, we first analyzed all of the trials within a given subject where a congruent audiovisual video was shown (and the reported percept was correct) and computed the average confidence for each subject. Next, we analyzed McGurk trials by selecting specific instances where the reported subject percept matched one of the congruent conditions (e.g., analyzing the "Na" answers from the McGurk condition, which could be compared with the answers from the "auditory $\mathrm{Na}$, visual $\mathrm{Na"} \mathrm{condition)} \mathrm{and} \mathrm{computing} \mathrm{the}$ average confidence for these trials, too. As shown in Figure 3A, the average confidence for congruent audiovisual syllables (91.8) was higher than the average confidence for McGurk videos with matched syllable content (81.8). To test whether this difference was significant, we conducted a Shapiro-Wilk test of normality, which suggested a deviation from normality $(\mathrm{W}=$ $0.90, p=.005)$. Considering the non-normality of our data, we then conducted a Wilcoxon Signed-Rank test, which indicated that the average confidence in congruent audiovisual videos was significantly higher than average confidence in the McGurk videos with matched syllable content $(W=518.00, p<.001)$. 

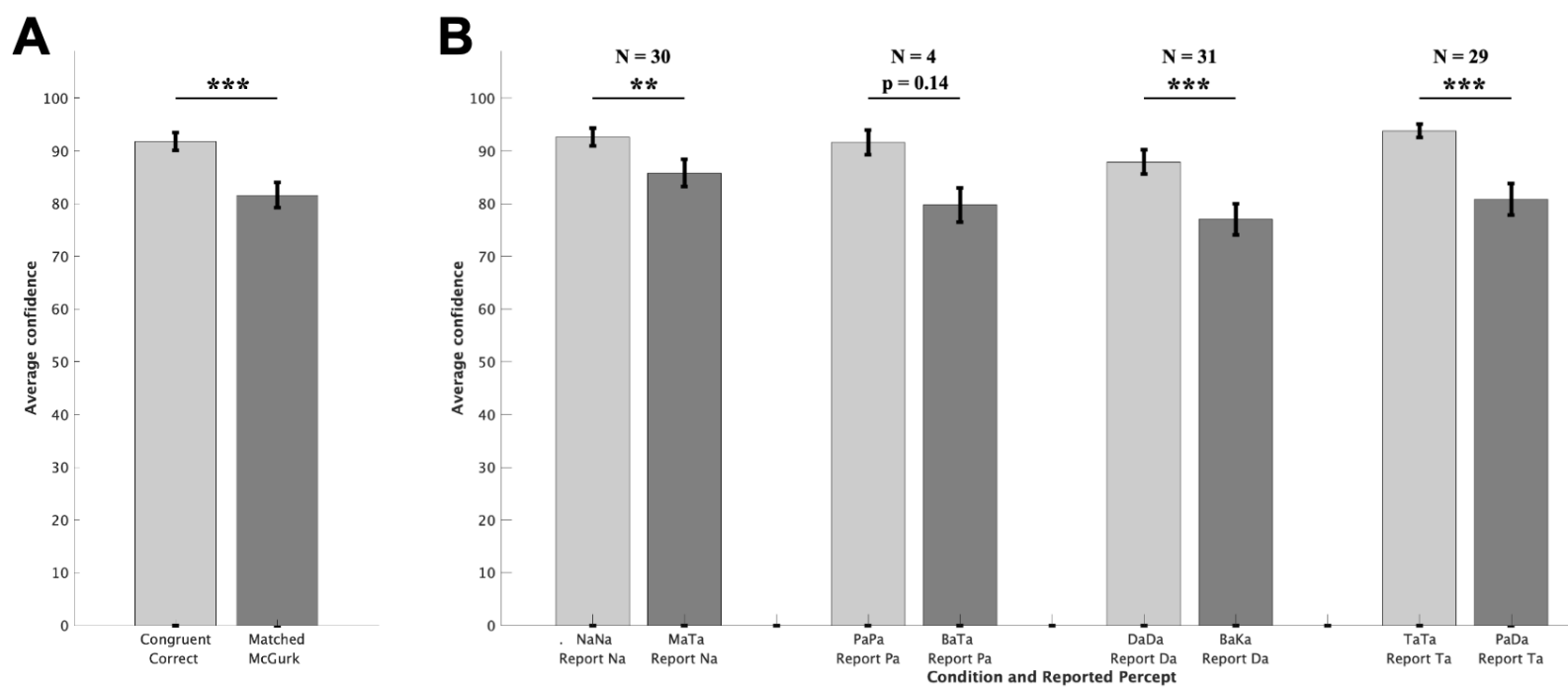

Figure 3. Confidence in audiovisual speech perception for matched syllables. (A) Confidence for Congruent and McGurk conditions with identical reported percepts, averaged across all four audiovisual syllable combinations. "Congruent correct" refers to trials where congruent audiovisual videos were shown, and the participants correctly perceived the syllable. "Matched McGurk" refers to trials where a McGurk stimulus was shown, and the reported syllable matched the reported syllable in the corresponding "congruent" condition. Shown here are results for average confidence (averaged across all subjects) for both of these conditions, with error bars showing the S.E.M. across subjects. (B) Average confidence for each specific matched syllable-report condition. Shown here is the confidence (averaged across all subjects) for specific audiovisual congruent conditions when a correct report was given (e.g., auditory $\mathrm{Na}$ /visual $\mathrm{Na}$ - perceive $\mathrm{Na}$ ) and trials from McGurk conditions with a specific type of report that matched a congruent condition (e.g., auditory Ma/visual Ta, but the subject reported $\mathrm{Na}$, etc.). Since not all subjects reported the McGurk syllable that matched the syllable from the congruent condition, above the graph, we report the number of subjects that had at least one trial with matched syllable content. For three of the four conditions, matched content was common, but for the BaTa condition, reported "Pa" syllables were quite rare in this sample. Error bars represent S.E.M. across subjects. ${ }^{* * *} p<.001,{ }^{* *} p<.01$

Further analysis revealed the robustness of this effect. Figure 3B shows the average confidence for each of the four matched-content conditions. As can be seen in the figure, across all four conditions, the average confidence was higher for the audiovisual congruent condition compared to trials from the corresponding McGurk condition with a matched syllable report. To determine whether the differences in confidence between each condition were significant or not, we conducted post-hoc tests, which revealed that confidence was significantly higher in three of the four audiovisual congruent conditions $(\mathrm{Na}: t(29)=3.05, p<.005$; Da: $t(30)=4.16, p<0.001$; Ta: $\mathrm{t}(28)=5.77, \mathrm{p}<.001 ; \mathrm{Pa:} \mathrm{t}(3)=1.96, \mathrm{p}=0.14)$. Because there were two answer choices on 
each McGurk trial that could be considered "fused," (see Table 1, Stropahl et al., 2017) this paradigm carried the risk of not producing matched syllable content. While three of our four conditions were quite robust in producing high numbers of subjects with matched syllable content, we did notice that one condition was deficient in this sample: for auditory Ba-visual Ta, perceiving the McGurk syllable "Pa" was a relative rarity, with only 4 out of 32 subjects producing matched syllable content. This contrasted the robust matching in the other three conditions, with each condition producing matched syllable content in at least 29 out of the 32 subjects.

Overall, the rates of how often participants perceived "fused" stimuli in the McGurk conditions was $71.9 \%$, with fusion rates ranging between $62 \%$ and $80 \%$ for any specific condition. This fusion rate is in line with previous reports using these videos (Stropahl et al., 2017). The next most common responses in the McGurk trials were to report the auditory component of what was shown (25.6\%), and the least common response was to report the visual component of the video $(2.4 \%)$.

\section{Experiment 3 - Method}

In Experiment 3, we aimed to determine whether confidence would differ between McGurk speech and rarely integrated speech where the reported percept was identical (Deroy et al., 2016). While Experiment 2 provided evidence that confidence distinguished between congruent and illusory McGurk speech with matched percepts, both of these conditions are characterized by the same causal scenario (Figure 1). Thus, it remains an open question whether metacognitive systems distinguish between different types of multisensory stimuli when the causal structure differs across stimuli with matched reports (Deroy et al., 2016; Shams and Beierholm, 2021). 


\section{Participants}

Thirty participants enrolled in this online experiment, which was coded in jsPsych 6.3.1 (de Leeuw, 2015), and launched through the website Cognition.run. Participants were recruited through Prolific.co and were awarded $\$ 4.30$ upon completion of the task. All participants successfully completed the task and no data was excluded; thus, thirty participants were included in our final analysis (24 women, 6 men; mean age $=34.3$ years).

\section{Stimuli}

For our McGurk stimuli in Experiment 3, we again utilized video and audio clips from the Oldenburg Audio Visual Speech Stimuli (OLAVS) (Stropahl et al., 2017) and created six audiovisual combinations from Speaker TK01. We had two conditions: McGurk stimuli and "Rarely Integrated" stimuli, with each condition consisting of three unique audiovisual combinations. The McGurk stimuli pairs included auditory Ma/visual Ta, auditory Ba/visual Ka, and auditory Pa/visual Da. The "rarely integrated" incongruent stimulus pairs included auditory $\mathrm{Na/visual} \mathrm{Da,} \mathrm{auditory} \mathrm{Da/visual} \mathrm{Ma,} \mathrm{and} \mathrm{auditory} \mathrm{Ta/visual} \mathrm{Ga.} \mathrm{Our} \mathrm{motivation} \mathrm{for} \mathrm{selecting}$ these specific stimuli was to create conditions with matched reported percepts between the McGurk and rarely integrated conditions, to evaluate how metacognition tracks perception. Thus, the anticipated perceived syllables for each of the three McGurk and rarely integrated conditions were "Na," "Da," and "Ta," respectively. For McGurk trials, the possible answer choices that were shown to subjects corresponded to the visual syllable, the auditory syllable, and two syllables which reflect "fused" percepts (from Stropahl et al., 2017). For rarely integrated trials, the possible answer choices corresponded to the visual syllable, the auditory syllable, and two "foil" answers that did not correspond to any presented or integrated percept.

\section{Procedure}

As in Experiment 1, participants began our online task by enrolling through the website Prolific.co. Following a welcome screen, participants read through our online consent form (IRB \#201902462, University of Florida) and provided consent by checking a box next to the 
statement, "I agree to participate in this study." Next, participants viewed an example photo which demonstrated how they should sit roughly one arm's length away from the screen. Following this, each participant's web camera was activated and they needed to ensure that their face was within view of the camera. After reporting their sex and age, participants were shown a sample 2-second McGurk stimulus video, and instructed that they could adjust the volume to a comfortable level for the experiment. Participants could repeat the video as many times as necessary as they adjusted the volume.

Next, participants completed six practice trials, viewing each of the six stimuli one time. On each trial, participants reported which syllable they perceived (selected from one of four possible options), and then rated their confidence in this report by moving a slider on a continuous scale from 0 (no confidence) to 100 (fully confident). Once the practice trials finished, participants began the main experiment, which consisted of 2 blocks of 60 trials (120 trials totals). Each of the 3 McGurk stimuli were presented 20 times each in the experiment; each of the three rarely integrated stimuli were presented 20 times each in the experiment. As in the practice session, participants reported the syllable on each trial, and also rated their confidence on the 0 to 100 scale. Upon completing the task, participants were given a completion code and were re-routed to Prolific.co to receive payment.

\section{Results}

Across subjects, the average confidence in all McGurk trials (mean $=84.2$ ) was quite similar to the average confidence in all rarely integrated trials (mean $=86.4)(t(29, p=0.13, d=$ 0.28). However, the primary analysis of interest in this experiment was to investigate differences between McGurk and rarely integrated syllables for matched percepts. To begin our main analysis, we first selected all of the rarely integrated and McGurk trials in each subject which produced the desired percepts: $\mathrm{Na}$, Da, and Ta. Next, we computed the average confidence for these trials. As shown in Figure 4A, on average across subjects, the mean confidence in rarely integrated trials was 88.1 , and the mean confidence in McGurk trials was 
85.1. A paired-sample t-test revealed that this difference was not significant $(t(29)=1.57, p=$ $0.13, d=0.29)$

A

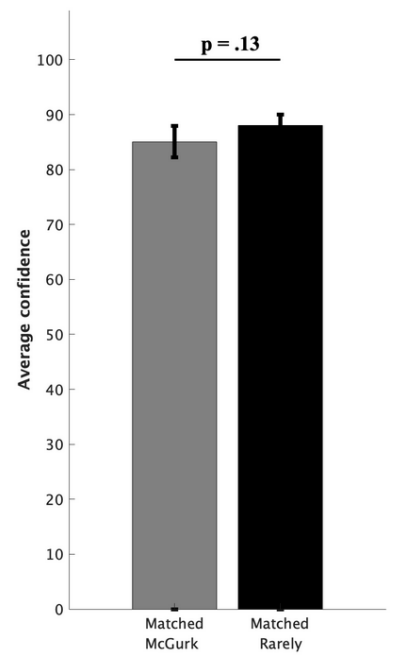

B

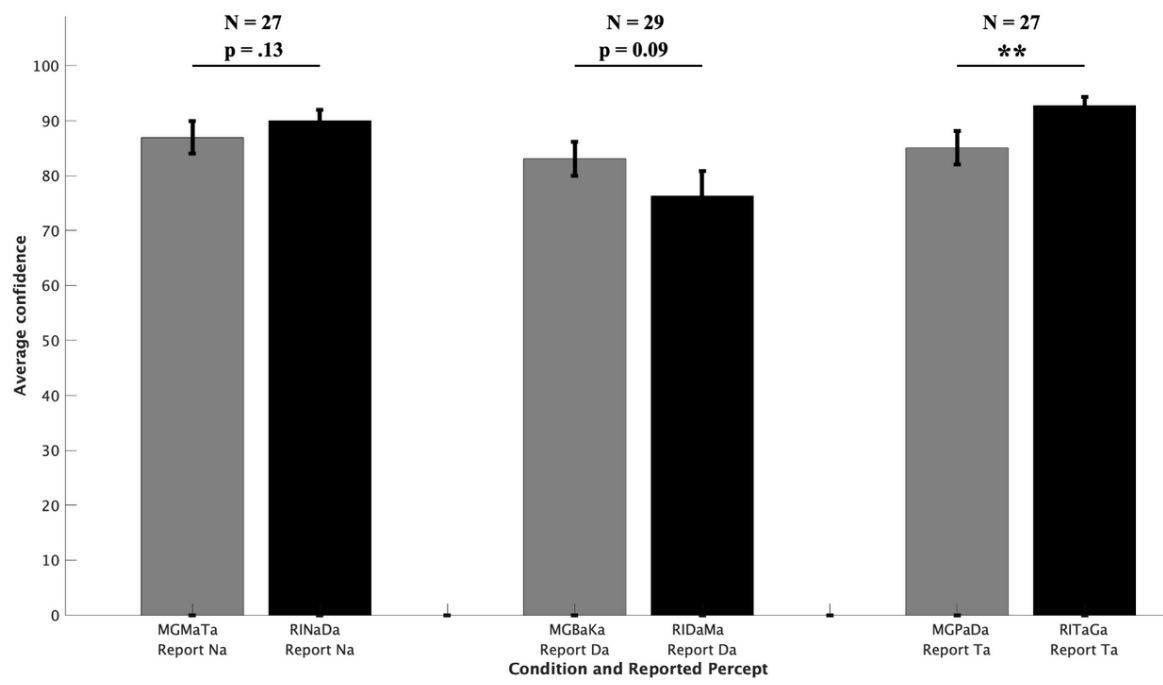

Figure 4. Confidence in audiovisual speech with different causal structures and matched syllable percepts. (A) Confidence for Congruent and McGurk conditions across, averaged across all four audiovisual syllable combinations. "Matched McGurk" refers to trials where a McGurk stimulus was shown, and the reported syllable matched the reported syllable in the corresponding "rarely integrated" condition. Shown here are results for average confidence (averaged across all subjects) for both of these conditions, with error bars showing the S.E.M. across subjects. (B) Average confidence for each specific matched syllable-report condition. Shown here is the confidence (averaged across all subjects) for specific audiovisual conditions when a specific syllable $(\mathrm{Na}$, Da, or $\mathrm{Ta})$ was reported. $\mathrm{MG}=\mathrm{McGurk} ; \mathrm{RI}=$ Rarely Integrated, and auditory syllables are listed first, with visual syllables listed second. Since not all subjects reported the McGurk syllable that matched the syllable from the congruent condition, above the graph, we report the number of subjects that had at least one trial with matched syllable content. Error bars represent S.E.M. across subjects. ${ }^{* *} p<.01$

To better understand whether any of our three specific condition pairings produced an effect, we analyzed each pair of conditions separately. First, we analyzed the pair of conditions that produced the percept "Na." As shown in Figure 4B, on trials where subjects reported "Na," the average confidence was slightly higher in the rarely integrated condition (auditory Na- visual $\mathrm{Da}$, Mean $=90.5$ ) compared to the McGurk condition (auditory Ma-visual Ta, mean $=87.0$ ), but this difference was not significant $(\mathrm{t}(26)=1.58, \mathrm{p}=0.13, d=0.30)$. On trials where subjects reported "Da," interestingly, the confidence was slightly higher in the McGurk condition (auditory 
$\mathrm{Ba}$ - visual Ka, Mean = 83.1) compared to the rarely integrated condition (auditory Da - visual Ma, Mean $=76.3)$, although again, this difference was not significant $(\mathrm{t}(28)=1.76, p=0.09, d=$ 0.33). Interestingly, in our final condition where subjects reported "Ta," confidence was higher in the rarely integrated condition (auditory $\mathrm{Ta}$ - visual $\mathrm{Ga}$, mean $=92.8$ ) compared to the rarely integrated condition (auditory $\mathrm{Pa}$ - visual $\mathrm{Da}$, mean $=85.1$ ), and this difference was significant $(t(26)=2.87, p<0.01, d=0.55)$. Together, these results provide evidence that metacognition's ability to distinguish between causal scenarios with matched percepts may not be defined by a general trend which holds across all stimulus types, and may instead depend on the specific stimuli that are perceived.

\section{Discussion}

In this investigation, we aimed to answer two questions: can metacognition tease apart differences between congruent, integrated, and segregated audiovisual speech? And can it do so when the reported percepts are the same? Using McGurk speech stimuli (Stropahl et al., 2017), in our first experiment, results showed that for unmatched percepts, confidence judgments were significantly higher for audiovisual congruent stimuli compared to McGurk stimuli. Interestingly, stimuli that were rarely integrated produced an intermediate level of confidence, which was higher than the McGurk trials, but lower than congruent trials. In our second experiment, when the perceptual content was matched between congruent and McGurk trials, confidence was higher for each congruent condition compared to the matched McGurk percept. Our third experiment showed that confidence in perceived syllables for rarely integrated trials and McGurk trials with matched percepts were quite similar, without a clear trend. In one condition, confidence was higher in the rarely integrated trials, but in the other two conditions, confidence was not significantly different. These results show that it might be challenging for confidence judgments to distinguish between different causal structures when the percept is matched, and that if they can, the effect might be stimulus-specific. 
As previous research has noted (Deroy et al., 2016), little is currently known about the relationship between metacognition and multisensory integration (Garzorz and Deroy, 2020). One previous investigation provided preliminary evidence that confidence differs between different forms of audiovisual speech: in a study of speech perception in schizophrenia, agematched controls exhibited lower confidence (on average) with McGurk speech, compared to fully congruent or incongruent audiovisual speech (White et al., 2014). Results from our first experiment replicated this finding. While the work by (White et al., 2014) provided preliminary evidence that metacognition could distinguish between stimuli that are produced by different causal structures (Körding et al., 2007), questions about whether or not metacognition can distinguish between stimuli that yield matched reports with different underlying causal structures, or access the underlying unisensory cues in different contexts, had remained unanswered (Deroy et al., 2016; Shams and Beierholm, 2021). Here, we provide evidence that when reported percepts are identical, metacognition can distinguish between congruent and McGurk speech, but that it may not be able to distinguish between matched percepts generated by distinct causal structures.

One question that is not answered by the current investigation is whether our findings here would generalize to paradigms where the sensory signals obtained by observers are less salient. Specifically, multisensory speech stimuli include signals that are easy to perceive; the observer's lips and voice, at least in this study, are not particularly "noisy" sources of information (Bishop and Miller, 2009). Whether our findings would generalize to paradigms that include more ambiguous stimuli remains to be seen. For example, stimuli that are frequently used to study spatial and temporal interactions in multisensory paradigms are often much more ambiguous (Alais and Burr, 2004; Bertelson and Radeau, 1976; Chen and Vroomen, 2013; Ernst and Banks, 2002; Parise et al., 2012; Shams et al., 2000; Welch and Warren, 1980). Thus, future studies should aim to evaluate whether results from these experiments replicate in other multisensory paradigms, including speech paradigms which use different syllable 
combinations than the ones employed in this study. We have preliminary data which indicate that metacognition distinguishes matched percepts in the sound-induced flash illusion (Mims and Odegaard, 2020), but much more work is needed in this area.

Overall, we think these results can inform recent work which characterizes perceptual awareness as a higher-order state in generative models of perceptual contents (Fleming, 2020). Specifically, while the Bayesian Causal Inference models that have dominated multisensory research for the last decade and a half have produced profound insights into both behavioral (Körding et al., 2007; Samad et al., 2015) and neural (Rideaux et al., 2021; Rohe et al., 2019; Rohe and Noppeney, 2015) correlates of multisensory integration, linking Bayesian models to sensory phenomenology and sensory awareness has proven extremely difficult (Denison et al., 2020). Our results provide preliminary evidence that perceptual awareness may be characterized by knowledge of which signals in the environment are truly congruent, and which signals in the environment represent an integrated, "best guess" at the current state of the world. Considering the mixed nature of the results in Experiment 3, we recommend that future research aim to explore the degree to which metacognition may (or may not) be able to distinguish the internal and external origins of sensory causes. 


\section{Author Contributions}

Victoria Cardenas coded Experiment 1 and assisted with data collection using Google Drive's webAPI and subject payments via Prolific.co. Samantha Pedersen coded Experiment 2 and assisted with data collection using Google Drive's webAPI and subject payments via Prolific.co. Faith Kimmet coded Experiment 3, assisted with data collection on Cognition.run and subject payments via Prolific.co. Grey Johnson assisted with the online implementation of Experiments 1, 2, and 3. Brian Odegaard coded the analyses for Experiments 1-3. Brian Odegaard, Faith Kimmet, and Addison Sans wrote the manuscript jointly, which was proofread and endorsed by all authors. Upon manuscript acceptance, all code and analyses for this project will be released on the Open Science Framework. 


\section{References}

Alais D and Burr D (2004) The ventriloquist effect results from near-optimal bimodal integration. Current biology: CB 14(3): 257-262.

Bertelson P and Radeau M (1976) Ventriloquism, sensory interaction, and response bias: Remarks on the paper by Choe, Welch, Gilford, and Juola. Perception \& psychophysics 19(6): 531-535.

Bishop CW and Miller LM (2009) A multisensory cortical network for understanding speech in noise. Journal of cognitive neuroscience 21(9). direct.mit.edu: 1790-1805.

Chen L and Vroomen J (2013) Intersensory binding across space and time: a tutorial review. Attention, perception \& psychophysics 75(5): 790-811.

de Leeuw JR (2015) jsPsych: a JavaScript library for creating behavioral experiments in a Web browser. Behavior research methods 47(1): 1-12.

Denison RN, Block N and Samaha J (2020) What do models of visual perception tell us about visual phenomenology? DOI: 10.31234/osf.io/7p8jg.

Deroy O, Spence C and Noppeney U (2016) Metacognition in Multisensory Perception. Trends in cognitive sciences 20(10): 736-747.

Ernst MO and Banks MS (2002) Humans integrate visual and haptic information in a statistically optimal fashion. Nature 415(6870): 429-433.

Flavell JH (1979) Metacognition and cognitive monitoring: A new area of cognitivedevelopmental inquiry. The American psychologist 34(10): 906-911.

Fleming SM (2020) Awareness as inference in a higher-order state space. Neuroscience of consciousness 2020(1): niz020.

Fleming SM and Lau HC (2014) How to measure metacognition. Frontiers in human neuroscience 8: 443.

Freeman J and Simoncelli EP (2011) Metamers of the ventral stream. Nature neuroscience 14(9): 1195-1201.

Garzorz I and Deroy O (2020) Why There Is a Vestibular Sense, or How Metacognition Individuates the Senses. Multisensory research 34(3): 261-280.

Knill DC and Richards W (1996) Perception as Bayesian Inference. Cambridge University Press.

Körding KP, Beierholm U, Ma WJ, et al. (2007) Causal inference in multisensory perception. PloS one 2(9): e943.

Magnotti JF and Beauchamp MS (2017) A Causal Inference Model Explains Perception of the McGurk Effect and Other Incongruent Audiovisual Speech. PLoS computational biology 13(2): e1005229.

Magnotti JF, Ma WJ and Beauchamp MS (2013) Causal inference of asynchronous audiovisual 
speech. Frontiers in psychology 4: 798.

Magnotti JF, Smith KB, Salinas M, et al. (2018) A causal inference explanation for enhancement of multisensory integration by co-articulation. Scientific reports 8(1): 18032.

McGovern DP, Roudaia E, Newell FN, et al. (2016) Perceptual learning shapes multisensory causal inference via two distinct mechanisms. Scientific reports 6: 24673.

McGurk H and MacDonald J (1976) Hearing lips and seeing voices. Nature 264(5588). nature.com: 746-748.

Mims CE and Odegaard B (2020) Metacognitive Awareness in the Sound-Induced Flash Illusion. DOI: 10.31234/osf.io/5yexg.

Odegaard B, Wozny DR and Shams L (2015) Biases in Visual, Auditory, and Audiovisual Perception of Space. PLoS computational biology 11(12): e1004649.

Parise CV, Spence C and Ernst MO (2012) When correlation implies causation in multisensory integration. Current biology: $C B$ 22(1): 46-49.

Pick HL, Warren DH and Hay JC (1969) Sensory conflict in judgments of spatial direction. Perception \& psychophysics 6(4). Springer: 203-205.

Rahnev D, Balsdon T, Charles L, et al. (2021) Consensus goals for the field of visual metacognition. DOI: 10.31234/osf.io/z8v5x.

Rideaux R, Storrs KR, Maiello G, et al. (2021) How multisensory neurons solve causal inference. Proceedings of the National Academy of Sciences of the United States of America 118(32). DOI: 10.1073/pnas.2106235118.

Rohe T and Noppeney U (2015) Cortical hierarchies perform Bayesian causal inference in multisensory perception. PLoS biology 13(2): e1002073.

Rohe T, Ehlis A-C and Noppeney U (2019) The neural dynamics of hierarchical Bayesian causal inference in multisensory perception. Nature communications 10(1): 1907.

Samad M, Chung AJ and Shams L (2015) Perception of body ownership is driven by Bayesian sensory inference. PloS one 10(2): e0117178.

Shams L and Beierholm U (2021) Bayesian Causal Inference: A Unifying Neuroscience Theory. DOI: $10.31234 / o s f . i o / x p z 6 n$.

Shams L and Beierholm UR (2010) Causal inference in perception. Trends in cognitive sciences 14(9): 425-432.

Shams L, Kamitani Y and Shimojo S (2000) Illusions. What you see is what you hear. Nature 408(6814): 788.

Stevenson RA, Zemtsov RK and Wallace MT (2012) Individual differences in the multisensory temporal binding window predict susceptibility to audiovisual illusions. Journal of experimental psychology. Human perception and performance 38(6): 1517-1529.

Stropahl M, Schellhardt S and Debener S (2017) McGurk stimuli for the investigation of 
multisensory integration in cochlear implant users: The Oldenburg Audio Visual Speech Stimuli (OLAVS). Psychonomic bulletin \& review 24(3): 863-872.

van Erp JBF, Philippi TG and Werkhoven P (2013) Observers can reliably identify illusory flashes in the illusory flash paradigm. Experimental brain research. Experimentelle Hirnforschung. Experimentation cerebrale 226(1): 73-79.

Welch RB and Warren DH (1980) Immediate perceptual response to intersensory discrepancy. Psychological bulletin 88(3): 638-667.

White TP, Wigton RL, Joyce DW, et al. (2014) Eluding the illusion? Schizophrenia, dopamine and the McGurk effect. Frontiers in human neuroscience 8. frontiersin.org: 565. 